\title{
COMPLETE SPACELIKE SUBMANIFOLDS IN DE SITTER SPACES WITH $R=a H+b$
}

\author{
JIANCHENG LIU ${ }^{\otimes}$ and JINGJING ZHANG
}

(Received 14 March 2012; accepted 20 November 2012; first published online 13 February 2013)

\begin{abstract}
In this paper, we give a classification of spacelike submanifolds with parallel normalised mean curvature vector field and linear relation $R=a H+b$ of the normalised scalar curvature $R$ and the mean curvature $H$ in the de Sitter space $S_{p}^{n+p}(c)$.
\end{abstract}

2010 Mathematics subject classification: primary 53C42; secondary 53C15.

Keywords and phrases: de Sitter space, spacelike submanifold, parallel normalised mean curvature vector, totally umbilical, hyperbolic cylinder.

\section{Introduction}

Let $\mathbb{R}_{p}^{n+p}$ denote an $(n+p)$-dimensional real vector space endowed with an inner product of index $p$ given by

$$
\langle x, y\rangle=-\sum_{i=1}^{p} x_{i} y_{i}+\sum_{j=p+1}^{n+p} x_{j} y_{j}
$$

where $x=\left(x_{1}, x_{2}, \ldots, x_{n+p}\right)$ are the natural coordinates of $\mathbb{R}_{p}^{n+p}$. The manifold $\mathbb{R}_{p}^{n+p}$ is called semi-Euclidean space and it has constant curvature $c=0$. We also define the semi-Riemannian manifold $S_{p}^{n+p}(c)$, with $c>0$, the so-called de Sitter space, as follows:

$$
S_{p}^{n+p}(c)=\left\{\left(x_{1}, x_{2}, \ldots, x_{n+p}\right) \in \mathbb{R}_{p}^{n+p+1}:-\sum_{i=1}^{p} x_{i}^{2}+\sum_{j=p+1}^{n+p+1} x_{j}^{2}=\frac{1}{c}\right\} .
$$

A smooth immersion $\phi: M^{n} \rightarrow S_{p}^{n+p}(c)$ of an $n$-dimensional connected manifold $M^{n}$ is said to be a spacelike submanifold if the induced metric via $\phi$ is a Riemannian metric on $M^{n}$. When the codimension $p=1$, it is called a spacelike hypersurface.

Spacelike submanifolds usually appear in the study of questions related to causality in general relativity. More precisely, level sets of a function of global time are

This work was financially supported by National Natural Science Foundation of China (Grant no. 11261051, 11171246), and Fundamental Research Funds of the Gansu Universities.

(C) 2013 Australian Mathematical Publishing Association Inc. 0004-9727/2013 \$16.00 
spacelike submanifolds. Also, spacelike hypersurfaces with constant mean curvature are convenient as initial hypersurfaces for the Cauchy problem in an arbitrary spacetime and for studying the propagation of gravitational radiation.

The initial step for the study of spacelike submanifolds in the de Sitter space $S_{p}^{n+p}(c)$ is due to Goddard's conjecture [11]: complete spacelike hypersurfaces with constant mean curvature $H$ in $S_{1}^{n+1}(1)$ must be totally umbilical. It was proved by Akutagawa [2] (for the case where $n=2$ and $H^{2} \leq 1$ or $n \geq 3$ and $H^{2}<4(n-1) / n^{2}$ ) and by Montiel [15] (for the compact case) that the conjecture is true. Furthermore, Montiel [15] exhibited examples of complete spacelike hypersurfaces with constant $H$ satisfying $H^{2} \geq 4(n-1) / n^{2}$ but not umbilical-the so-called hyperbolic cylinders, which are isometric to the Riemannian product $H^{1}(\sinh r) \times S^{n-1}(\cosh r)$.

Having completely settled Goddard's conjecture, most of the research interest turned to the study of submanifolds in $S_{p}^{n+p}(c)$ with constant scalar curvature instead of constant mean curvature. In particular, interest focuses on characterising the totally umbilical properties or Riemannian product structures (that is, hyperbolic cylinders) of such submanifolds. We refer to $[5,6,9,14,19]$ and the references therein.

More generally, Cheng [8], Li [13] and Shu [17] studied the spacelike hypersurface $M$ in $S_{1}^{n+1}(1)$ under the condition that the normalised scalar curvature $R$ and the mean curvature $H$ of $M$ satisfy the linear relation $R=a H$ for some constant $a$, instead of the restriction that $R$ or $H$ is a constant. Such a spacelike hypersurface $M$ is called a Weingarten hypersurface. They obtained many sufficient conditions for such a Weingarten hypersurface to be totally umbilical. Recently, Hou and Yang [12] extended the linear relation $R=a H$ to the case $R=a H+b$ for some constants $a, b$, and generalised the corresponding results of Cheng [8, Theorem 1] (when $b=0$ and $a$ is positive), Li [13, Corollary 4.3] (when $b=0$ ) and Zheng [19, Theorem B] (when $a=0)$.

In this paper, we will study the situation of higher codimension under the more general relation $R=a H+b$, and prove the following two theorems.

Theorem 1.1. Let $M^{n}(n \geq 2)$ be a complete spacelike submanifold in the de Sitter space $S_{p}^{n+p}(c)$ with parallel normalised mean curvature vector. Assume that the normalised scalar curvature $R$ and the mean curvature $H$ of $M^{n}$ satisfy $R=a H+b$ for some constants $a \neq 0$ and $b<c$, and $H$ attains a maximum on $M^{n}$. If the squared norm $S$ of the second fundamental form of $M^{n}$ satisfies $S \leq 2 \sqrt{n-1} c$, then either $M^{n}$ is totally umbilical or $S=2 \sqrt{n-1} c$ and $M^{n}$ is isometric to a hyperbolic cylinder $H^{1}(\sinh r) \times S^{n-1}(\cosh r)$.

Theorem 1.2. Let $M^{n}(n \geq 2)$ be a complete spacelike submanifold in the de Sitter space $S_{p}^{n+p}(c)$ with parallel normalised mean curvature vector. Assume that the normalised scalar curvature $R$ and the mean curvature $H$ of $M^{n}$ satisfy $R=a H+b$ for some constants $a \neq 0$ and $b<c$, and $H$ attains a maximum on $M^{n}$.

(1) When $H^{2} \leq c$ for $n=2$ or $H^{2}<4(n-1) c /\left((n-2)^{2} p+4(n-1)\right)$ for $n \geq 3$, then $M^{n}$ is totally umbilical. 
(2) When $H^{2}=4(n-1) c /\left((n-2)^{2} p+4(n-1)\right)$ for $n \geq 3$, then $M^{n}$ is totally umbilical, or the codimension $p=1$ and $M^{n}$ is isometric to a hyperbolic cylinder $H^{1}(\sinh r) \times S^{n-1}(\cosh r)$.

(3) When $4(n-1) c /\left((n-2)^{2} p+4(n-1)\right)<H^{2} \leq c$ for $n \geq 3$, if the squared norm $S$ of the second fundamental form of $M^{n}$ satisfies $S \leq n H^{2}+\left(B_{H}^{-}\right)^{2}$ or $S \geq$ $n H^{2}+\left(B_{H}^{+}\right)^{2}$, then $M^{n}$ is totally umbilical, or the codimension $p=1$ and $M^{n}$ is isometric to a hyperbolic cylinder $H^{1}(\sinh r) \times S^{n-1}(\cosh r)$, where $B_{H}^{ \pm}$are the two real roots of the polynomial

$$
P_{H}(x)=\frac{1}{p} x^{2}-\frac{n(n-2)}{\sqrt{n(n-1)}} H x+n\left(c-H^{2}\right) .
$$

REMARK 1.3. When the constant $a$ vanishes identically, the linear relation $R=a H+b$ reduces to $R=b$, that is, $R$ is a constant. Therefore we restrict our attention to $a \neq 0$.

REMARK 1.4. The parallel normalised mean curvature vector assumption was introduced by Chen [7]. Submanifolds with nonzero parallel mean curvature vector also have parallel normalised mean curvature vector. The condition of having a parallel normalised mean curvature vector is much weaker than the condition of having a parallel mean curvature vector. For instance, every hypersurface in a semi-Riemannian manifold always has a parallel normalised mean curvature vector. In this sense, Theorem 1.1, for $p=1$, generalises [12, Theorem 1.5], and Theorem 1.2 reduces to the result of [17] when the constant $b$ vanishes identically and $a$ is positive.

REMARK 1.5. In our main Theorems 1.1 and 1.2, we assume that the mean curvature $H$ attains a maximum on the submanifold $M^{n}$. Use of the Cheng-Yau operator, first used in the same sense by Cheng in [8], and then by Hou and Yang in [12], is clearly a helpful technical condition to impose. We do not yet know whether it is necessary. In other words, we do not have an example of a spacelike hypersurface with parallel mean curvature vector for which the maximum of the mean curvature may occur at infinity.

REMARK 1.6. In fact, the results in Theorem 1.2 partially concern the reduction of codimensions, which is an important problem in the classification of submanifolds. When the ambient spaces are Riemannian space forms, we refer to Barbosa and Araújo's work [4]; the references therein include the classical results. But as we know, there are few results in semi-Riemannian space forms.

\section{Preliminaries}

Let $S_{p}^{n+p}(c)$ be an $(n+p)$-dimensional de Sitter space of constant sectional curvature $c>0$, whose index is $p$, and let $M^{n}$ be an $n$-dimensional complete spacelike submanifold immersed in $S_{p}^{n+p}(c)$. Throughout this paper, we shall make use of the convention on the range of indices:

$$
1 \leq A, B, \ldots \leq n+p ; \quad 1 \leq i, j, \ldots \leq n ; \quad n+1 \leq \alpha, \beta, \ldots \leq n+p .
$$


Choose a local field of semi-Riemannian orthonormal frames $\left\{e_{1}, \ldots, e_{n+p}\right\}$ such that, restricted to $M^{n},\left\{e_{1}, \ldots, e_{n}\right\}$ spans the tangent space of $M^{n}$ and forms an orthonormal frame there. Let $\left\{\omega_{1}, \ldots, \omega_{n+p}\right\}$ be its dual frame field so that the semiRiemannian metric of $S_{p}^{n+p}(c)$ is given by $d \bar{s}^{2}=\sum_{A} \varepsilon_{A} \omega_{A}^{2}$, where $\varepsilon_{i}=1, \varepsilon_{\alpha}=-1$. Then the structure equations of $S_{p}^{n+p}(c)$ are given by

$$
\begin{gathered}
d \omega_{A}=\sum_{B} \varepsilon_{B} \omega_{A B} \wedge \omega_{B}, \quad \omega_{A B}+\omega_{B A}=0, \\
d \omega_{A B}=\sum_{C} \varepsilon_{C} \omega_{A C} \wedge \omega_{C B}-\frac{1}{2} \sum_{C, D} \varepsilon_{C} \varepsilon_{D} K_{A B C D} \omega_{C} \wedge \omega_{D}, \\
K_{A B C D}=c \varepsilon_{A} \varepsilon_{B}\left(\delta_{A C} \delta_{B D}-\delta_{A D} \delta_{B C}\right) .
\end{gathered}
$$

Restricting these forms to $M^{n}$, then $\omega_{\alpha}=0$. By Cartan's lemma, we can write $\omega_{\alpha i}=$ $\sum_{j} h_{i j}^{\alpha} \omega_{j}, h_{i j}^{\alpha}=h_{j i}^{\alpha}$. The connection forms of $M^{n}$ are characterised by the structure equations

$$
\begin{aligned}
d \omega_{i} & =\sum_{j} \omega_{i j} \wedge \omega_{j}, \quad \omega_{i j}+\omega_{j i}=0, \\
d \omega_{i j} & =\sum_{k} \omega_{i k} \wedge \omega_{k j}-\frac{1}{2} \sum_{k, l} R_{i j k l} \omega_{k} \wedge \omega_{l},
\end{aligned}
$$

and

$$
R_{i j k l}=c\left(\delta_{i k} \delta_{j l}-\delta_{i l} \delta_{j k}\right)-\sum_{\alpha}\left(h_{i k}^{\alpha} h_{j l}^{\alpha}-h_{i l}^{\alpha} h_{j k}^{\alpha}\right),
$$

where $R_{i j k l}$ are the components of the curvature tensor of $M^{n}$. Denote by $R_{i k}$, $n(n-1) R$ and $R_{\alpha \beta i j}$ the components of the Ricci curvature, the scalar curvature and the components of normal curvature of $M^{n}$, respectively. Then, from (2.1),

$$
\begin{gathered}
R_{i j}=c(n-1) \delta_{i j}-\sum_{\alpha, k} h_{k k}^{\alpha} h_{i j}^{\alpha}+\sum_{\alpha, k} h_{i k}^{\alpha} h_{k j}^{\alpha}, \\
n(n-1) R=n(n-1) c+S-n^{2} H^{2}, \\
R_{\alpha \beta i j}=\sum_{l}\left(h_{i l}^{\alpha} h_{j l}^{\beta}-h_{j l}^{\alpha} h_{i l}^{\beta}\right) .
\end{gathered}
$$

Recall that $h=\sum_{\alpha, i, j} h_{i j}^{\alpha} \omega_{i} \otimes \omega_{j} \otimes e_{\alpha}$ is the second fundamental form; then the mean curvature vector $\vec{H}$, its length $H$, and the squared length $S$ of $h$ are defined by

$$
\vec{H}=\frac{1}{n} \sum_{\alpha, i} h_{i i}^{\alpha} e_{\alpha}, \quad H=\frac{1}{n} \sqrt{\sum_{\alpha}\left(\sum_{i} h_{i i}^{\alpha}\right)^{2}}, \quad S=\sum_{\alpha, i, j}\left(h_{i j}^{\alpha}\right)^{2} .
$$

Define the first and the second covariant derivatives of $h_{i j}^{\alpha}$, say $h_{i j k}^{\alpha}$ and $h_{i j k l}^{\alpha}$, by

$$
\begin{gathered}
\sum_{k} h_{i j k}^{\alpha} \omega_{k}=d h_{i j}^{\alpha}+\sum_{k} h_{j k}^{\alpha} \omega_{k i}+\sum_{k} h_{i k}^{\alpha} \omega_{k j}-\sum_{\beta} h_{i j}^{\beta} \omega_{\beta \alpha}, \\
\sum_{m} h_{i j k m}^{\alpha} \omega_{m}=d h_{i j k}^{\alpha}+\sum_{m} h_{m j k}^{\alpha} \omega_{m i}+\sum_{m} h_{i m k}^{\alpha} \omega_{m j}+\sum_{m} h_{i j m}^{\alpha} \omega_{m k}-\sum_{\beta} h_{i j k}^{\beta} \omega_{\beta \alpha} .
\end{gathered}
$$


We obtain the Codazzi equations and the Ricci identities

$$
\begin{gathered}
h_{i j k}^{\alpha}=h_{i k j}^{\alpha}=h_{j i k}^{\alpha}, \\
h_{i j k l}^{\alpha}-h_{i j l k}^{\alpha}=\sum_{m} h_{m i}^{\alpha} R_{m j k l}+\sum_{m} h_{m j}^{\alpha} R_{m i k l}+\sum_{\beta} h_{i j}^{\beta} R_{\alpha \beta k l} .
\end{gathered}
$$

The Laplacian of $h_{i j}^{\alpha}$ is defined by $\Delta h_{i j}^{\alpha}=\sum_{k} h_{i j k k}^{\alpha}$. It follows from (2.4) that, for any $\alpha$,

$$
\Delta h_{i j}^{\alpha}=\sum_{k} h_{k k i j}^{\alpha}+\sum_{k, m} h_{i m}^{\alpha} R_{m k j k}+\sum_{k, m} h_{k m}^{\alpha} R_{m i j k}+\sum_{k, \beta} h_{i k}^{\beta} R_{\alpha \beta j k} .
$$

Since the normalised mean curvature vector field is parallel, we choose $\vec{H}=H e_{n+1}$, then $n H=\sum_{i} h_{i i}^{n+1}$, and for any $\alpha>n+1, \sum_{i} h_{i i}^{\alpha}=0$. Denote by $H^{\alpha}$ the matrix $\left(h_{i j}^{\alpha}\right)$. Then

$$
\operatorname{tr}\left(H^{n+1}\right)=n H, \quad \operatorname{tr}\left(H^{\alpha}\right)=0 \quad \text { for } \alpha \geq n+2 .
$$

Set $\Phi=\sum_{\alpha, i, j} \Phi_{i j}^{\alpha} \omega_{i} \otimes \omega_{j} \otimes e_{\alpha}$, where $\Phi_{i j}^{n+1}=h_{i j}^{n+1}-H \delta_{i j}$, and for any $\alpha \geq n+2, \Phi_{i j}^{\alpha}=$ $h_{i j}^{\alpha}$. Let $\Phi^{\alpha}$ denote the matrix $\left(\Phi_{i j}^{\alpha}\right)$. Then

$$
\Phi^{n+1}=H^{n+1}-H I, \quad \Phi^{\alpha}=H^{\alpha} \quad \text { for } \alpha \geq n+2,
$$

and

$$
\left|\Phi^{n+1}\right|^{2}=\operatorname{tr}\left(H^{n+1}\right)^{2}-n H^{2}, \quad \sum_{\alpha>n+1}\left|\Phi^{\alpha}\right|^{2}=\sum_{\alpha>n+1}\left(h_{i j}^{\alpha}\right)^{2}, \operatorname{tr}\left(\Phi^{\alpha}\right)=0, \quad \forall \alpha .
$$

So we arrive at

$$
S=\sum_{\alpha}\left|\Phi^{\alpha}\right|^{2}+n H^{2}=|\Phi|^{2}+n H^{2}
$$

and $\frac{1}{2} \Delta S=\sum_{\alpha, i, j, k}\left(h_{i j k}^{\alpha}\right)^{2}+\sum_{\alpha, i, j} h_{i j}^{\alpha} \Delta h_{i j}^{\alpha}$. When the normalised mean curvature vector field of $M^{n}$ is parallel, using (2.1), (2.3), (2.5) and (2.6), a direct calculation yields (see [6])

$$
\begin{gathered}
\frac{1}{2} \Delta S=\sum_{\alpha, i, j, k}\left(h_{i j k}^{\alpha}\right)^{2}+n \sum_{\alpha, i, j} h_{i j}^{\alpha} H_{i j}^{\alpha}+n c\left(S-n H^{2}\right)-n H \sum_{\alpha} \operatorname{tr}\left(H^{n+1}\left(H^{\alpha}\right)^{2}\right) \\
+\sum_{\alpha, \beta}\left(\operatorname{tr}\left(H^{\alpha} H^{\beta}\right)\right)^{2}+\sum_{\alpha, \beta} N\left(H^{\alpha} H^{\beta}-H^{\beta} H^{\alpha}\right)
\end{gathered}
$$

where $N(A)=\operatorname{tr}\left(A A^{\prime}\right)$ for an $n \times n$ matrix $A$, with $A^{\prime}$ its transpose. By (2.7)-(2.9) we can obtain

$$
\begin{gathered}
n c S-n^{2} c H^{2}=n c\left(|\Phi|^{2}+n H^{2}\right)-n^{2} c H^{2}=n c|\Phi|^{2}, \\
n H \sum_{\alpha} \operatorname{tr}\left(H^{n+1}\left(H^{\alpha}\right)^{2}\right)=n H \sum_{\alpha} \operatorname{tr}\left(\Phi^{n+1}\left(\Phi^{\alpha}\right)^{2}\right)+2 n H^{2} \operatorname{tr}\left(\Phi^{n+1}\right)^{2} \\
+n^{2} H^{4}+n H^{2}|\Phi|^{2}, \\
\sum_{\alpha, \beta}\left(\operatorname{tr}\left(H^{\alpha} H^{\beta}\right)\right)^{2}=\sum_{\alpha, \beta}\left(\operatorname{tr}\left(\Phi^{\alpha} \Phi^{\beta}\right)\right)^{2}+n^{2} H^{4}+2 n H^{2} \operatorname{tr}\left(\Phi^{n+1}\right)^{2} .
\end{gathered}
$$


Following Cheng and Yau [10], we introduce the second-order operator $\square$, acting on any $C^{2}$ function $f$ on $M^{n}$,

$$
\square f=\sum_{i, j}\left(n H \delta_{i j}-h_{i j}^{n+1}\right) f_{i j} .
$$

We will need the following algebraic lemmas.

Lemma 2.1 [3]. Let $\left\{\mu_{i}\right\}_{i=1}^{n}$ be a set of real numbers satisfying $\sum_{i} \mu_{i}=0, \sum_{i} \mu_{i}^{2}=\beta^{2}$, $\beta \geq 0$. Then

$$
\left|\sum_{i} \mu_{i}^{3}\right| \leq \frac{n-2}{\sqrt{n(n-1)}} \beta^{3},
$$

and equality holds if and only if at least $n-1$ of the $\mu_{i}$ are equal to each other.

Lemma 2.2 [16]. Let $A, B: \mathbb{R}^{n} \rightarrow \mathbb{R}^{n}$ be two symmetric linear maps such that $A B-$ $B A=0$ and $\operatorname{tr}(A)=\operatorname{tr}(B)=0$. Then

$$
\left|\operatorname{tr}\left(A^{2} B\right)\right| \leq \frac{n-2}{\sqrt{n(n-1)}} N(A) \sqrt{N(B)} .
$$

Lemma 2.3 [18]. Let $a_{1}, \ldots, a_{n}, b_{1}, \ldots, b_{n}$ be $2 n(n \geq 2)$ real numbers satisfying $\sum_{i} b_{i}=0$. Then

$$
\sum_{i, j} a_{i} a_{j}\left(b_{i}-b_{j}\right)^{2} \leq \frac{n}{\sqrt{n-1}} \sum_{i} a_{i}^{2} \sum_{j} b_{j}^{2} .
$$

Since the parallel normalised mean curvature vector assumption implies that $\omega_{n+1 \alpha}=0$ for all $\alpha$, it is possible to show that $H^{n+1} H^{\alpha}=H^{\alpha} H^{n+1}$, for all $\alpha$ (see [6]). Therefore, by definition, the traceless matrix $\Phi^{n+1}$ commutes with the traceless matrices $\Phi^{\alpha}$, for all $\alpha$. Hence we can apply Lemma 2.2 and the Cauchy-Schwarz inequality in order to obtain, respectively,

$$
\begin{gathered}
\sum_{\alpha} \operatorname{tr}\left(\Phi^{n+1}\left(\Phi^{\alpha}\right)^{2}\right) \leq \frac{n-2}{\sqrt{n(n-1)}} \sqrt{N\left(\Phi^{n+1}\right)}|\Phi|^{2} \leq \frac{n-2}{\sqrt{n(n-1)}}|\Phi|^{3}, \\
\sum_{\alpha, \beta}\left(\operatorname{tr}\left(\Phi^{\alpha} \Phi^{\beta}\right)\right)^{2} \\
\geq \sum_{\alpha}\left(N\left(\Phi^{\alpha}\right)\right)^{2} \geq \frac{1}{p}\left(\sum_{\alpha} N\left(\Phi^{\alpha}\right)\right)^{2} \geq \frac{1}{p}|\Phi|^{4} .
\end{gathered}
$$

\section{Some lemmas}

To prove the main theorems, we need the following key lemmas.

Lemma 3.1. Let $M^{n}$ be an n-dimensional spacelike submanifold immersed in the de Sitter space $S_{p}^{n+p}(c)$ with $R=a H+b$. If $a \neq 0$ and $b<c$, then the operator $L=\square+\frac{1}{2}(n-1) a \triangle$ is elliptic. 
Proof. If $H=0$, then $R=b$. It follows from (2.2) that $S=n(n-1)(b-c)<0$, which is impossible. Therefore $H>0$. From (2.2) and $R=a H+b$, we know that

$$
S=n^{2} H^{2}+n(n-1)(a H+b-c),
$$

and then

$$
a=\frac{1}{n(n-1) H}\left(S-n^{2} H^{2}+n(n-1)(c-b)\right) .
$$

For any fixed $\alpha$, we choose locally an appropriate orthonormal frame $\left\{e_{j}\right\}$ such that $h_{i j}^{\alpha}=\lambda_{i}^{\alpha} \delta_{i j}$. Then $n H=\sum_{i} h_{i i}^{n+1}$ and $\sum_{i} h_{i i}^{\alpha}=0$ for any $\alpha \geq n+2$. For any $i$,

$$
\begin{aligned}
n H & -\lambda_{i}^{n+1}+\frac{n-1}{2} a \\
& =\sum_{j} \lambda_{j}^{n+1}-\lambda_{i}^{n+1}+\frac{1}{2 n H}\left(S-n^{2} H^{2}+n(n-1)(c-b)\right) \\
& \geq \sum_{j} \lambda_{j}^{n+1}-\lambda_{i}^{n+1}+\frac{1}{2}\left(\sum_{j}\left(\lambda_{j}^{n+1}\right)^{2}-\left(\sum_{j} \lambda_{j}^{n+1}\right)^{2}+n(n-1)(c-b)\right)(n H)^{-1} \\
& =\left(\left(\sum_{j} \lambda_{j}^{n+1}\right)^{2}-\lambda_{i}^{n+1} \sum_{j} \lambda_{j}^{n+1}-\frac{1}{2} \sum_{l \neq j} \lambda_{l}^{n+1} \lambda_{j}^{n+1}+\frac{1}{2} n(n-1)(c-b)\right)(n H)^{-1} \\
& =\left(\sum_{j \neq i}\left(\lambda_{j}^{n+1}\right)^{2}+\frac{1}{2} \sum_{l \neq j, l, j \neq i} \lambda_{l}^{n+1} \lambda_{j}^{n+1}+\frac{1}{2} n(n-1)(c-b)\right)(n H)^{-1} \\
& =\frac{1}{2}\left(\sum_{j \neq i}\left(\lambda_{j}^{n+1}\right)^{2}+\left(\sum_{j \neq i} \lambda_{j}^{n+1}\right)^{2}+n(n-1)(c-b)\right)(n H)^{-1} .
\end{aligned}
$$

It follows from $b<c$ that $n H-\lambda_{i}^{n+1}+\frac{1}{2}(n-1) a>0$. Thus $L$ is an elliptic operator. This completes the proof of Lemma 3.1.

Lemma 3.2. Let $M^{n}$ be an n-dimensional spacelike submanifold in the de Sitter space $S_{p}^{n+p}(c)$ with $R=a H+b$. If $a \neq 0$ and $b<c$, then

$$
\sum_{i, j, k, \alpha}\left(h_{i j k}^{\alpha}\right)^{2} \geq n^{2}|\nabla H|^{2}
$$

Proof. Taking the covariant derivative of (3.1), for any $k$,

$$
2 \sum_{i, j, \alpha} h_{i j}^{\alpha} h_{i j k}^{\alpha}=\left(2 n^{2} H+n(n-1) a\right) H_{k} .
$$

Hence, by the Cauchy-Schwarz inequality,

$$
\sum_{i, j, \alpha}\left(h_{i j}^{\alpha}\right)^{2} \sum_{i, j, k, \alpha}\left(h_{i j k}^{\alpha}\right)^{2} \geq\left(n^{2} H+\frac{1}{2} n(n-1) a\right)^{2}|\nabla H|^{2} .
$$


That is,

$$
S \sum_{i, j, k, \alpha}\left(h_{i j k}^{\alpha}\right)^{2} \geq\left(n^{2} H+\frac{1}{2} n(n-1) a\right)^{2}|\nabla H|^{2} .
$$

On the other hand, it follows from (3.1) that

$$
\begin{aligned}
\left(n^{2} H+\frac{1}{2} n(n-1) a\right)^{2}-n^{2} S & =n^{2}\left(n^{2} H^{2}+n(n-1) H a-S\right)+\frac{1}{4} n^{2}(n-1)^{2} a^{2} \\
& =n^{3}(n-1)(c-b)+\frac{1}{4} n^{2}(n-1)^{2} a^{2} \\
& =\frac{1}{4} n^{2}(n-1)\left((n-1) a^{2}+4 n(c-b)\right) .
\end{aligned}
$$

Since $a \neq 0$ and $b<c$, we have $\left(n^{2} H+\frac{1}{2} n(n-1) a\right)^{2}>n^{2} S$. Hence

$$
S \sum_{i, j, k, \alpha}\left(h_{i j k}^{\alpha}\right)^{2} \geq\left(n^{2} H+\frac{1}{2} n(n-1) a\right)^{2}|\nabla H|^{2} \geq n^{2} S|\nabla H|^{2},
$$

which implies that $S=0$, or $\sum_{i, j, k, \alpha}\left(h_{i j k}^{\alpha}\right)^{2} \geq n^{2}|\nabla H|^{2}$.

Lemma 3.3. Let $M^{n}$ be an $n$-dimensional complete spacelike submanifold immersed in the de Sitter space $S_{p}^{n+p}(c)$ with parallel normalised mean curvature vector field. Then

$$
\square(n H) \geq-\frac{1}{2} n(n-1) \Delta R+\left(S-n H^{2}\right)\left(n c-\frac{n}{2 \sqrt{n-1}} S\right) .
$$

Proof. By the definition of the operator $\square$,

$$
\begin{aligned}
\square(n H) & =\sum_{i, j}\left(n H \delta_{i j}-h_{i j}^{n+1}\right)(n H)_{i j} \\
& =\frac{1}{2} \Delta\left(n^{2} H^{2}\right)-n^{2}|\nabla H|^{2}-\sum_{i, j} h_{i j}^{n+1}(n H)_{i j} \\
& =\frac{1}{2} \Delta(S-n(n-1)(R-c))-n^{2}|\nabla H|^{2}-\sum_{i, j} h_{i j}^{n+1}(n H)_{i j} \\
& =-\frac{1}{2} n(n-1) \triangle R+\frac{1}{2} \triangle S-n^{2}|\nabla H|^{2}-\sum_{i, j} h_{i j}^{n+1}(n H)_{i j} .
\end{aligned}
$$

Let

$$
\|\mu\|^{2}=\sum_{i, j}\left(h_{i j}^{n+1}-H \delta_{i j}\right)^{2}, \quad\|\tau\|^{2}=\sum_{i, j} \sum_{\alpha>n+1}\left(h_{i j}^{\alpha}\right)^{2} .
$$

It is clear that the functions $\|\mu\|^{2}$ and $\|\tau\|^{2}$ are defined on $M^{n}$ globally; in other words, they do not depend on the choice of the orthonormal frame $\left\{e_{1}, \ldots, e_{n}\right\}$. Also,

$$
S=\|\mu\|^{2}+\|\tau\|^{2}+n H^{2}
$$


which leads to

$$
\frac{1}{2} \Delta S=\frac{1}{2} \Delta\|\mu\|^{2}+\frac{1}{2} \Delta\|\tau\|^{2}+\frac{1}{2} \Delta\left(n H^{2}\right)=\frac{1}{2} \Delta \operatorname{tr}\left(H^{n+1}\right)^{2}+\frac{1}{2} \Delta\|\tau\|^{2} .
$$

Together with (2.1), (2.3) and (2.5),

$$
\begin{aligned}
\frac{1}{2} \Delta \operatorname{tr}\left(H^{n+1}\right)^{2}= & \sum_{i, j, k}\left(h_{i j k}^{n+1}\right)^{2}+\sum_{i, j} h_{i j}^{n+1} \Delta h_{i j}^{n+1} \\
= & \sum_{i, j, k}\left(h_{i j k}^{n+1}\right)^{2}+\sum_{i, j} h_{i j}^{n+1}(n H)_{i j}+n c \operatorname{tr}\left(H^{n+1}\right)^{2}-n^{2} c H^{2} \\
& \quad-n H \operatorname{tr}\left(H^{n+1}\right)^{3}+\left(\operatorname{tr}\left(H^{n+1}\right)^{2}\right)^{2}+\sum_{\beta>n+1}\left(\operatorname{tr}\left(H^{n+1} H^{\beta}\right)\right)^{2}
\end{aligned}
$$

Choose an appropriate orthonormal frame such that $h_{i j}^{n+1}=\lambda_{i} \delta_{i j}$. Since $\sum_{i}\left(\lambda_{i}-H\right)=0$, then

$$
\begin{aligned}
& \sum_{i}\left(\lambda_{i}-H\right)^{2}=\sum_{i} \lambda_{i}^{2}-n H^{2}=\operatorname{tr}\left(H^{n+1}\right)^{2}-n H^{2}=\|\mu\|^{2} \\
& \sum_{i} \lambda_{i}^{3}=\sum_{i}\left(\lambda_{i}-H\right)^{3}+n H^{3}+3 H\|\mu\|^{2}
\end{aligned}
$$

By Lemma 2.1,

$$
\begin{aligned}
-n H \operatorname{tr}\left(H^{n+1}\right)^{3} & =-n^{2} H^{4}-3 n H^{2}\|\mu\|^{2}-n H \sum_{i}\left(\lambda_{i}-H\right)^{3} \\
& \geq-n^{2} H^{4}-3 n H^{2}\|\mu\|^{2}-\frac{n(n-2)}{\sqrt{n(n-1)}} H\|\mu\|^{3} .
\end{aligned}
$$

Putting the above three formulas into (3.5),

$$
\begin{aligned}
\frac{1}{2} \Delta \operatorname{tr}\left(H^{n+1}\right)^{2} \geq \sum_{i, j, k}\left(h_{i j k}^{n+1}\right)^{2}+\sum_{i, j} h_{i j}^{n+1}(n H)_{i j} \\
+\|\mu\|^{2}\left(\|\mu\|^{2}-\frac{n(n-2)}{\sqrt{n(n-1)}} H\|\mu\|+n\left(c-H^{2}\right)\right) .
\end{aligned}
$$

Consider the quadratic form

$$
F(x, y)=x^{2}-\frac{n-2}{\sqrt{n-1}} x y-y^{2} .
$$

By the orthogonal transformation

$$
\begin{aligned}
& u=\frac{1}{\sqrt{2 n}}((1+\sqrt{n-1}) x+(1-\sqrt{n-1}) y), \\
& v=\frac{1}{\sqrt{2 n}}((\sqrt{n-1}-1) x+(\sqrt{n-1}+1) y),
\end{aligned}
$$


it is clear that $x^{2}+y^{2}=u^{2}+v^{2}$ and $F(x, y)=n\left(u^{2}-v^{2}\right) / 2 \sqrt{n-1}$. Taking $x=\|\mu\|$, $y=\sqrt{n H^{2}}$, we obtain $u^{2}+v^{2}=x^{2}+y^{2}=\|\mu\|^{2}+n H^{2}$. Hence

$$
\begin{aligned}
n c+F(x, y) & =n c-\frac{n}{2 \sqrt{n-1}}\left(u^{2}+v^{2}\right)+\frac{n}{\sqrt{n-1}} u^{2} \\
& \geq n c-\frac{n}{2 \sqrt{n-1}}\left(\|\mu\|^{2}+n H^{2}\right) \\
& \geq n c-\frac{n}{2 \sqrt{n-1}} S,
\end{aligned}
$$

which implies that

$$
n c+\|\mu\|^{2}-n H^{2}-\frac{n(n-2)}{\sqrt{n(n-1)}} H\|\mu\| \geq n c-\frac{n}{2 \sqrt{n-1}} S .
$$

Together with (3.6),

$$
\frac{1}{2} \Delta \operatorname{tr}\left(H^{n+1}\right)^{2} \geq \sum_{i, j, k}\left(h_{i j k}^{n+1}\right)^{2}+\sum_{i, j} h_{i j}^{n+1}(n H)_{i j}+\|\mu\|^{2}\left(n c-\frac{n}{2 \sqrt{n-1}} S\right) .
$$

For any $\alpha>n+1$,

$$
-n H \operatorname{tr}\left(\left(H^{\alpha}\right)^{2} H^{n+1}\right)+\left(\operatorname{tr}\left(H^{n+1} H^{\alpha}\right)\right)^{2}=-\frac{1}{2} \sum_{i, j} h_{i i}^{n+1} h_{j j}^{n+1}\left(h_{i i}^{\alpha}-h_{j j}^{\alpha}\right)^{2} .
$$

Let $a_{i}=h_{i i}^{n+1}, b_{i}=h_{i i}^{\alpha}$. Since $\sum_{i} h_{i i}^{\alpha}=0, \alpha>n+1$, by Lemma 2.3,

$$
-n H \operatorname{tr}\left(\left(H^{\alpha}\right)^{2} H^{n+1}\right)+\left(\operatorname{tr}\left(H^{n+1} H^{\alpha}\right)\right)^{2} \geq-\frac{n}{2 \sqrt{n-1}}\left(\sum_{j}\left(h_{j j}^{\alpha}\right)^{2}\right)\left(\sum_{i}\left(h_{i i}^{n+1}\right)^{2}\right) .
$$

Taking the sum on both sides with respect to $\alpha>n+1$,

$$
-n H \sum_{\alpha>n+1} \operatorname{tr}\left(\left(H^{\alpha}\right)^{2} H^{n+1}\right)+\sum_{\alpha>n+1}\left(\operatorname{tr}\left(H^{n+1} H^{\alpha}\right)\right)^{2} \geq-\frac{n}{2 \sqrt{n-1}}\|\tau\|^{2} S .
$$

Then, using (2.1), (2.3) and (2.5),

$$
\begin{aligned}
\frac{1}{2} \Delta\|\tau\|^{2}= & \sum_{i, j, k, \alpha>n+1}\left(h_{i j k}^{\alpha}\right)^{2}+n c\|\tau\|^{2}+\sum_{\alpha>n+1}\left(\operatorname{tr}\left(H^{\alpha} H^{n+1}\right)\right)^{2} \\
& +\sum_{\alpha, \beta>n+1}\left(\operatorname{tr}\left(H^{\alpha} H^{\beta}\right)\right)^{2}-n H \sum_{\alpha>n+1}\left(\operatorname{tr}\left(H^{\alpha}\right)^{2} H^{n+1}\right) \\
& +\sum_{\alpha, \beta>n+1} N\left(H^{\alpha} H^{\beta}-H^{\beta} H^{\alpha}\right) .
\end{aligned}
$$

Since $\sum_{\alpha, \beta>n+1}\left(\operatorname{tr}\left(H^{\alpha} H^{\beta}\right)\right)^{2} \geq 0$ and $\sum_{\alpha, \beta>n+1} N\left(H^{\alpha} H^{\beta}-H^{\beta} H^{\alpha}\right) \geq 0$, from (3.9) and (3.10)

$$
\frac{1}{2} \Delta\|\tau\|^{2} \geq \sum_{i, j, k, \alpha>n+1}\left(h_{i j k}^{\alpha}\right)^{2}+\|\tau\|^{2}\left(n c-\frac{n}{2 \sqrt{n-1}} S\right) .
$$


Putting (3.8) and (3.11) into (3.2), and combining (3.3), (3.4) and Lemma 3.2, we complete the proof of Lemma 3.3.

\section{Proofs of main theorems}

Proof of Theorem 1.1. Applying Lemmas 3.1 and 3.3,

$$
\begin{aligned}
L(n H) & =\square(n H)+\frac{n-1}{2} a \triangle(n H)=\square(n H)+\frac{1}{2} n(n-1) \triangle R \\
& \geq\left(S-n H^{2}\right)\left(n c-\frac{n}{2 \sqrt{n-1}} S\right) .
\end{aligned}
$$

It is clear that $S \geq n H^{2}$ from (3.3). Together with the assumption $S \leq 2 \sqrt{n-1} c$ in Theorem 1.1, we obtain $L(n H) \geq 0$. Since $L$ is elliptic and $H$ obtains a maximum on $M^{n}$, we deduce that $H$ is a constant. Hence

$$
\left(S-n H^{2}\right)\left(n c-\frac{n}{2 \sqrt{n-1}} S\right)=0 .
$$

If $S<2 \sqrt{n-1} c$ then $S=n H^{2}$, and $M^{n}$ is totally umbilical.

If $S=2 \sqrt{n-1} c$, the inequalities in (3.7) and in Lemma 2.1 become equalities. If the inequalities in (3.7) hold, then $\|\tau\|^{2}=0$. Since $e_{n+1}$ is parallel on the normal bundle $T^{\perp}\left(M^{n}\right)$ of $M^{n}$, we know that $M^{n}$ lies in a totally geodesic submanifold $S_{1}^{n+1}(c)$ of $S_{p}^{n+p}(c)$. If equality holds in Lemma 2.1, then $M^{n}$ has $n-1$ principal curvatures which are equal and constant. As $H$ is a constant, the other principal curvature is a constant as well. So $M^{n}$ is isometric to a hyperbolic cylinder $H^{1}(\sinh r) \times S^{n-1}(\cosh r)$ from the congruence theorem in [1]. This completes the proof of Theorem 1.1.

Proof of Theorem 1.2. By the definition of operator $\square$, and using Lemma 3.2 and (2.10),

$$
\begin{aligned}
\square(n H)=- & \frac{1}{2} n(n-1) \triangle R+\frac{1}{2} \triangle S-n^{2}|\nabla H|^{2}-\sum_{i, j} h_{i j}^{n+1}(n H)_{i j} \\
=- & \frac{1}{2} n(n-1) \triangle R+\sum_{\alpha, i, j, k}\left(h_{i j k}^{\alpha}\right)^{2}+\sum_{i, j} h_{i j}^{n+1}(n H)_{i j}+n c\left(S-n H^{2}\right) \\
& -n H \sum_{\alpha} \operatorname{tr}\left(H^{n+1}\left(H^{\alpha}\right)^{2}\right)+\sum_{\alpha, \beta}\left(\operatorname{tr}\left(H^{\alpha} H^{\beta}\right)\right)^{2} \\
& +\sum_{\alpha, \beta} N\left(H^{\alpha} H^{\beta}-H^{\beta} H^{\alpha}\right)-n^{2}|\nabla H|^{2}-\sum_{i, j} h_{i j}^{n+1}(n H)_{i j} \\
\geq- & \frac{1}{2} n(n-1) \triangle R+n c\left(S-n H^{2}\right)-n H \sum_{\alpha} \operatorname{tr}\left(H^{n+1}\left(H^{\alpha}\right)^{2}\right) \\
& +\sum_{\alpha, \beta} N\left(H^{\alpha} H^{\beta}-H^{\beta} H^{\alpha}\right)+\sum_{\alpha, \beta}\left(\operatorname{tr}\left(H^{\alpha} H^{\beta}\right)\right)^{2} .
\end{aligned}
$$


Notice that $\sum_{\alpha, \beta} N\left(H^{\alpha} H^{\beta}-H^{\beta} H^{\alpha}\right) \geq 0$. Together with (2.11)-(2.13), then (4.1) becomes

$$
\begin{aligned}
\square(n H) \geq- & \frac{1}{2} n(n-1) \triangle R+\left(n c-n H^{2}\right)|\Phi|^{2} \\
& -n H \sum_{\alpha} \operatorname{tr}\left(\Phi^{n+1}\left(\Phi^{\alpha}\right)^{2}\right)+\sum_{\alpha, \beta}\left(\operatorname{tr}\left(\Phi^{\alpha} \Phi^{\beta}\right)\right)^{2} .
\end{aligned}
$$

Substituting (2.14), (2.15) into (4.2),

$$
\square(n H) \geq-\frac{1}{2} n(n-1) \triangle R+|\Phi|^{2}\left(\frac{|\Phi|^{2}}{p}-\frac{n(n-2)}{\sqrt{n(n-1)}} H|\Phi|+n\left(c-H^{2}\right)\right) .
$$

Furthermore, note that $R=a H+b$. Then

$$
\begin{aligned}
L(n H) & =\square(n H)+\frac{n-1}{2} a \triangle(n H)=\square(n H)+\frac{1}{2} n(n-1) \triangle R \\
& \geq|\Phi|^{2}\left(\frac{|\Phi|^{2}}{p}-\frac{n(n-2)}{\sqrt{n(n-1)}} H|\Phi|+n\left(c-H^{2}\right)\right) .
\end{aligned}
$$

Put

$$
P_{H}(|\Phi|)=\frac{|\Phi|^{2}}{p}-\frac{n(n-2)}{\sqrt{n(n-1)}} H|\Phi|+n\left(c-H^{2}\right) .
$$

(1) When $n=2$ and $H^{2} \leq c, P_{H}(|\Phi|) \geq 0$. Hence, the right-hand side of (4.3) is nonnegative. Since $L$ is elliptic and $H$ obtains a maximum on $M^{n}$, we deduce that $H$ is a constant and (4.3) becomes

$$
|\Phi|^{2}\left(\frac{|\Phi|^{2}}{p}+2\left(c-H^{2}\right)\right)=0
$$

which implies that $|\Phi|^{2}=0$ and $M^{n}$ is totally umbilical.

When $n \geq 3$ and $H^{2}<4(n-1) c /\left((n-2)^{2} p+4(n-1)\right)$, the discriminant of $P_{H}(|\Phi|)$ is negative. Then $P_{H}(|\Phi|)>0$ and

$$
L(n H) \geq|\Phi|^{2}\left(\frac{|\Phi|^{2}}{p}-\frac{n(n-2)}{\sqrt{n(n-1)}} H|\Phi|+n\left(c-H^{2}\right)\right) \geq 0 .
$$

Using again the facts that $L$ is elliptic and $H$ obtains a maximum on $M^{n}$, we deduce that $H$ is a constant and then (4.3) yields

$$
|\Phi|^{2}\left(\frac{|\Phi|^{2}}{p}-\frac{n(n-2)}{\sqrt{n(n-1)}} H|\Phi|+n\left(c-H^{2}\right)\right)=0 .
$$

Since $P_{H}(|\Phi|)>0$, we obtain that $|\Phi|^{2}=0$ and $M^{n}$ is totally umbilical.

(2) When $n \geq 3$ and $H^{2}=4(n-1) c /\left((n-2)^{2} p+4(n-1)\right)$, the discriminant of $P_{H}(|\Phi|)$ vanishes and $P_{H}(|\Phi|) \geq 0$. If $P_{H}(|\Phi|)>0$, by making use of the same assertion 
as in the proof of (1) above, we infer that $|\Phi|^{2}=0$ and $M^{n}$ is totally umbilical. If $P_{H}(|\Phi|)=0$, then the inequalities in (2.14) and (2.15) become equalities. That is,

$$
\sqrt{N\left(\Phi^{n+1}\right)}|\Phi|^{2}=|\Phi|^{3}, \quad|\Phi|^{4}=p \sum_{\alpha} N^{2}\left(\Phi^{\alpha}\right) .
$$

Since

$$
|\Phi|=\sqrt{\frac{c}{(n-2)^{2} p+4(n-1)}} \frac{n(n-2)}{\sqrt{n}} p>0,
$$

using (4.4) we get $N\left(\Phi^{n+1}\right)=|\Phi|^{2}$. On the one hand, by $|\Phi|^{2}=\sum_{\alpha} N\left(\Phi^{\alpha}\right)$ we have $N\left(\Phi^{\alpha}\right)=0, \alpha \geq n+2$, so $|\Phi|^{4}=p \sum_{\alpha}\left(N\left(\Phi^{\alpha}\right)\right)^{2}=p\left(N\left(\Phi^{n+1}\right)\right)^{2}=p|\Phi|^{4}$. Hence $p=1$. On the other hand, if equality holds in Lemma 2.1 , then $M^{n}$ has $n-1$ principal curvatures which are equal and constant. As $H$ is a constant, the other principal curvature is a constant as well. According to the congruence theorem due to Abe et al. [1], $M^{n}$ is isometric to a hyperbolic cylinder $H^{1}(\sinh r) \times S^{n-1}(\cosh r)$.

(3) When $n \geq 3$ and $4(n-1) c /\left((n-2)^{2} p+4(n-1)\right)<H^{2} \leq c$, the discriminant of $P_{H}(|\Phi|)$ is positive. Then $P_{H}(|\Phi|)$ has two real roots:

$$
B_{H}^{ \pm}=p \sqrt{\frac{n}{4(n-1)}}\left((n-2) H \pm \sqrt{\frac{\left((n-2)^{2} p+4(n-1)\right) H^{2}+4(n-1) c}{p}}\right)
$$

The assumption $S \leq n H^{2}+\left(B_{H}^{-}\right)^{2}$ or $S \geq n H^{2}+\left(B_{H}^{+}\right)^{2}$ on $M^{n}$ implies that $|\Phi| \leq B_{H}^{-}$or $|\Phi| \geq B_{H}^{+}$on $M^{n}$. Therefore we know that $P_{H}(|\Phi|) \geq 0$.

Clearly, $B_{H}^{+}$is always positive. On the other hand, $B_{H}^{-}>0$ if and only if $H^{2}<c$, while $B_{H}^{-}=0$ if and only if $H^{2}=c$.

When $4(n-1) c /\left((n-2)^{2} p+4(n-1)\right)<H^{2}<c$, we have $B_{H}^{-}>0$. If $S<n H^{2}+$ $\left(B_{H}^{-}\right)^{2}$ or $S>n H^{2}+\left(B_{H}^{+}\right)^{2}$, then we obtain $P_{H}(|\Phi|)>0$. By making use of the same assertion as in the proof of (1) above, we infer that $|\Phi|^{2}=0$ and $M^{n}$ is totally umbilical. If $S=n H^{2}+\left(B_{H}^{-}\right)^{2}$ or $S=n H^{2}+\left(B_{H}^{+}\right)^{2}$, then we obtain $P_{H}(|\Phi|)=0$. By the same arguments as in the proof of (2) above, we infer that $M^{n}$ is isometric to a hyperbolic cylinder $H^{1}(\sinh r) \times S^{n-1}(\cosh r)$.

When $4(n-1) c /\left((n-2)^{2} p+4(n-1)\right)<H^{2}=c$, we have $B_{H}^{-}=0$. If $S>n H^{2}+$ $\left(B_{H}^{+}\right)^{2}$, we obtain $P_{H}(|\Phi|)>0$; and if $S=n H^{2}+\left(B_{H}^{+}\right)^{2}$, we get $P_{H}(|\Phi|)=0$. The rest of the proof is the same as that in case (2), and this completes the proof of Theorem 1.2.

\section{Acknowledgement}

Both authors would like to take this opportunity to express their thanks to the referee for valuable suggestions which improved this paper.

\section{References}

[1] N. Abe, N. Koike and S. Yamaguchi, 'Congruence theorems for proper semi-Riemannian hypersurfaces in a real space form', Yokohama Math. J. 35 (1987), 123-136. 
[2] K. Akutagawa, 'On spacelike hypersurfaces with constant mean curvature in the de Sitter space', Math. Z. 196 (1987), 13-19.

[3] H. Alencar, M. do Carmo and A. G. Colares, 'Stable hypersurfaces with constant scalar curvature', Math. Z. 213 (1993), 117-131.

[4] E. R. Barbosa and K. O. Araújo, 'On complete submanifolds with bounded mean curvature', J. Geom. Phys. 61 (2011), 1957-1964.

[5] F. E. C. Camargo, R. M. B. Chaves and L. A. M. Sousa Jr, 'Rigidity theorems for complete spacelike hypersurfaces with constant scalar curvature in de Sitter space', Differ. Geom. Appl. 26 (2008), 592-599.

[6] F. E. C. Camargo, R. M. B. Chaves and L. A. M. Sousa Jr, 'New characterizations of complete spacelike submanifolds in semi-Riemannian space forms', Kodai Math. J. 32 (2009), 209-230.

[7] B. Y. Chen, 'Surfaces with parallel normalized mean curvature vector', Monatsh. Math. 90 (1980), 185-194.

[8] Q. M. Cheng, 'Complete space-like hypersurfaces of a de Sitter space with $r=k H$ ', Mem. Fac. Sci., Kyushu University, Ser. A 44 (1990), 67-77.

[9] Q. M. Cheng and S. Ishikawa, 'Spacelike hypersurfaces with constant scalar curvature', Manuscripta Math. 95 (1998), 499-505.

[10] S. Y. Cheng and S. T. Yau, 'Hypersurfaces with constant scalar curvature', Math. Ann. 225 (1977), 195-204.

[11] A. Goddard, 'Some remarks on the existence of spacelike hypersurfaces of constant mean curvature', Math. Proc. Cambridge Philos. Soc. 82 (1977), 489-495.

[12] Z. H. Hou and D. Yang, 'Linear Weingarten spacelike hypersurfaces in de Sitter space', Bull. Belg. Math. Soc. Simon Stevin 17 (2010), 769-780.

[13] H. Li, 'Global rigidity theorems of hypersurfaces', Ark. Mat. 35 (1997), 327-351.

[14] J. C. Liu and D. Y. Zhang, 'Gap phenomena for complete space-like submanifolds with constant scalar curvature in de Sitter spaces', Chinese Ann. Math. 29A(5) (2008), 689-696.

[15] S. Montiel, 'An integral inequality for compact spacelike hypersurfaces in the de Sitter space and applications to the case of constant mean curvature', Indiana Univ. Math. J. 37 (1988), 909-917.

[16] W. Santos, 'Submanifolds with parallel mean curvature vector in spheres', Tôhoku Math. J. 46 (1994), 403-415.

[17] S. C. Shu, 'Complete spacelike hypersurfaces in a de Sitter space', Bull. Aust. Math. Soc. 73 (2006), 9-16.

[18] J. F. Zhang, Submanifolds with Constant Scalar Curvature and the Harmonic Functions of Finsler Manifolds, PhD Thesis, Zhejiang University, Hangzhou, China, 2005.

[19] Y. Zheng, 'On space-like hypersurfaces in the de Sitter space', Ann. Glob. Anal. Geom. 13 (1995), $317-321$.

JIANCHENG LIU, College of Mathematics and Statistics, Northwest Normal University, Lanzhou 730070, PR China e-mail: liujc@nwnu.edu.cn

JINGJING ZHANG, College of Mathematics and Statistics, Northwest Normal University, Lanzhou 730070, PR China e-mail: zhangjingjing3749@163.com 